\title{
TRANSTORNOS ESOFÁGICOS
}

\author{
Peter J. KAHRILAS ${ }^{1}$, André J. P. M. SMOUT²
}

\section{SINTOMAS ESOFÁGICOS}

Apesar dos avanços em testes de diagnóstico para as doenças gastrointestinais (GI), uma cuidadosa história clínica continua a ser fundamental para a avaliação de um paciente com sintomas gastrointestinais superiores. A maioria dos pacientes que apresentam transtornos esofágicos terá uma doença relativamente leve e não fatal e, em muitos casos, uma história cuidadosamente obtida levará a um tratamento rápido e preciso.

Como alternativa, também é importante identificar os pacientes portadores de uma maior probabilidade de doença subjacente grave para que possam ser investigados e geridos de forma rápida. Como parte da história, os médicos devem inquirir sobre hábitos alimentares do paciente, bem como consumo de fumo e álcool. Alguns indivíduos podem experimentar sintomas apenas quando comem excessivamente, particularmente se feito tão tarde da noite, antes de ir para a cama.

Os principais sintomas esofágicos são quatro, cada um deles associado a um distúrbio funcional de esôfago, sendo: azia, dor no peito, disfagia e globus.

\section{Azia (pirose)}

Este é o sintoma de origem esofágica encontrado com maior frequência. Azia é caracterizada por um desconforto ou sensação de queimação atrás do esterno que irradia para o epigástrio e pescoço ${ }^{(1)}$. É um sintoma intermitente, mais comumente experimentado dentro de 60 min após refeição, durante o exercício ou enquanto estiver deitado ou reclinado. O desconforto é aliviado com água ou antiácido, mas com freqüência interfere nas atividades habituais. Dada a elevada prevalência do refluxo gastroesofágico (RGE), seu valor preditivo como diagnóstico é alto quando a azia é o sintoma dominante ou exclusivo. A associação é tão forte que, em tais casos, a terapia empírica para DRGE tornou-se uma estratégia aceita na presença deste sintoma ${ }^{(2)}$. No entanto, o termo "azia" é frequente e indevidamente usado e/ou referido como outros termos, tais como "indigestão" ou "regurgitação", obrigando o médico a esclarecer o significado pretendido.

\section{Dor torácica}

Este é um sintoma de esôfago surpreendentemente comum com características muito semelhantes à dor cardíaca, sendo esta discriminação muito difícil em alguns casos. Dado o potencial de morbidade e mortalidade associadas com a dor cardíaca, convém sempre ter atenção para esta opção antes da esofági$\mathrm{ca}^{(3)}$. Dor esofágica geralmente é descrita como uma sensação de pressão no meio do peito, irradiando-se para a mandíbula, costas ou braços. A etiologia exata da dor esofágica é desconhecida, mas é demasiado simplista interpretá-la como indicativa de espasmo esofágico ou outra manifestação de uma anormalidade contrátil.

Raramente tais indivíduos objetivamente têm espasmo e nenhuma correlação pode ser estabelecida entre pequenas alterações da contratilidade esofágica e eventos de dor. Mas, provavelmente, as semelhanças com a dor cardíaca resultam do fato de que os dois órgãos compartilham um plexo de nervos e as terminações nervosas da parede esofágica terem pouca capacidade discriminativa entre os estímulos ${ }^{(4)}$.

${ }^{1}$ Division of Gastroenterology, Department of Medicine, Feinberg School of Medicine, Northwestern University, Chicago, Illinois, USA; ${ }^{2}$ Department of Gastroenterology and Hepatology, Academic Medical Center, Amsterdam, The Netherlands.

Correspondência: Peter J. Kahrilas, MD, Division of Gastroenterology, Department of Medicine, Feinberg School of Medicine, Northwestern University, 676 N Saint Clair, Suite 1400, Chicago, Illinois 60611-2951, USA. E-mail: p-kahrilas@northwestern.edu 
Distensão esofágica ou mesmo estimulação química (por exemplo, com ácido) será frequentemente percebida como dor torácica ${ }^{(5,6)}$. Na verdade, a DRGE é sua causa mais comum que a dismotilidade esofágica ${ }^{(3)}$.

\section{Disfagia}

Este sintoma é relatado em algum grau em mais de $30 \%$ dos indivíduos com DRGE. Pode ser causado por estenose péptica, anel de Schatzki, disfunção peristáltica, ou simplesmente pela inflamação da mucosa associada à esofagite. A disfagia também ocorre na ausência de qualquer anormalidade identificável, caso em que é provável ser resultado de sensibilidade anormal ao movimento de bolus durante o peristaltismo. Disfagia esofágica é frequentemente descrita como uma sensação de comida "abrir" o caminho para baixo ou até mesmo da parada do alimento no tórax por um período prolongado. Distinções importantes são entre disfagia de alimento exclusivamente sólido ao invés de líquidos e sólidos, se é episódica ou disfagia constante e progressiva, ou disfagia não progressiva. A disfagia para líquidos, como também para os alimentos sólidos, sugere uma anormalidade motora do esôfago, como a acalásia ${ }^{(7)}$. Por outro lado, a disfagia a alimentos sólidos exclusivamente é sugestiva de uma anormalidade estrutural como estenose, anel de Schatzki ou tumor e sempre deve ser investigada. Se a disfagia é apenas episódica, sugere um comprometimento leve do lúmen esofágico. Estes indivíduos podem relatar sintomas discrepantes: ou percebem que não há impedimento ao trânsito de alimentos ou que estão completamente obstruídos a tal ponto de até mesmo os líquidos voltarem. Disfagia progressiva para comida sólida é um achado preocupante, especialmente quando em associação à perda de peso, sendo esta a apresentação clássica do câncer de esôfago. A percepção do paciente quanto ao local de parada do alimento no esôfago é grosseiramente imprecisa.

Cerca de $30 \%$ das obstruções esofágicas distais são percebidas como disfagia cervical, com frequência induzindo a solicitar uma avaliação de distúrbio de deglutição orofaríngea. Em tais casos, o esôfago pode geralmente ser identificado como o culpado devido à ausência de sintomas concomitantes, geralmente associados à disfagia orofaríngea, tais como aspiração, regurgitação nasofaríngea, tosse, sialorreia ou óbvio comprometimento neuromuscular.

\section{Sensação de Globus}

Este sintoma, rotulado como alternativa "globus hystericus", é a percepção de um nódulo ou plenitude na garganta sentidos independentemente da deglutição ${ }^{(8)}$. Na verdade, embora tais pacientes sejam frequentemente encaminhados para uma avaliação da disfagia, a sensação de globus muitas vezes é aliviada pela deglutição. Como está implícito pelo seu outro nome (globus hystericus), a sensação de globus muitas vezes ocorre no cenário de distúrbios de ansiedade ou em obsessivos-compulsivos $^{(9)}$. A experiência clínica ensina que tal sintoma é atribuível à DRGE em uma fração substancial de pacientes, embora essa experiência amplamente não seja refletida na literatura científica ${ }^{(10)}$.

\section{PIROSE RECORRENTE}

\section{Anamnese}

Advogada de 34 anos de idade é encaminhada ao gastroenterologista pelo seu médico de atenção primária em razão de sintomas de refluxo "resistente à terapia". Tem sofrido por cerca de 5 anos com episódios diários de pirose que responderam parcialmente ao tratamento com o inibidor de bomba de prótons (IBP), prescrito pelo seu médico de atenção primária. Endoscopia do trato gastrointestinal superior (EGD) realizada 3 anos antes não revelou sinais macroscópicos de esofagite, bem como de hérnia de hiato.

Quando o gastroenterologista obtém a história, fica claro que os episódios de dor retroesternal em queimação (Caixa 1, Figura 1) experimentados pela paciente, duram de 10 minutos a algumas horas, não apresentam relação temporal clara com as refeições e não são dependentes da postura. A paciente não sofre de regurgitação nem de outros tipos de dor no peito. Não há disfagia, odinofagia nem outros sinais clínicos de alarme (Caixa 2). O uso de omeprazol $40 \mathrm{mg}$ diariamente (Caixa 3) parece melhorar um pouco os sintomas, mas o resultado é descrito como insatisfatório, mesmo com uma dose de $40 \mathrm{mg}$ duas vezes ao dia (Caixa 4). A paciente solicita tratamento cirúrgico.

O gastroenterologista decide repetir a endoscopia do trato GI superior (Caixa 6), após o período de 2 semanas sem IBP. Na endoscopia não são observadas anormalidades macroscópicas (Caixa 7). Não são obtidas biópsias. São, então, realizadas pHmetria e impedanciometria esofágicas de 24 horas (Caixa 9) após a paciente interromper o omeprazol por 7 dias. A exposição esofágica ao ácido (Caixa 10 ) encontra-se na faixa normal (tempo com $\mathrm{pH}<4$ : ereto $3,2 \%$, supino $0 \%$, total $2,3 \%$ ). Durante o registro de 24 horas a paciente apresenta seis episódios do sintoma. Nenhum destes episódios é associado temporalmente ao início de um episódio de refluxo, seja ácido ou não-ácido, resultando em uma probabilidade de associação de sintoma (PAS) de $0 \%$ (Caixa 12). Antes da colocação do cateter de $\mathrm{pH} / \mathrm{im}$ pedância, foi realizado estudo manométrico para medir a distância do esfíncter esofágico inferior (EEI) para a narina (Caixa 13). Durante este exame foi observada peristalse esofágica normal bem como pressão e relaxamento normal do EEI em repouso (Caixa 14). Conclui-se pelo diagnóstico de pirose funcional (Caixa 16). 


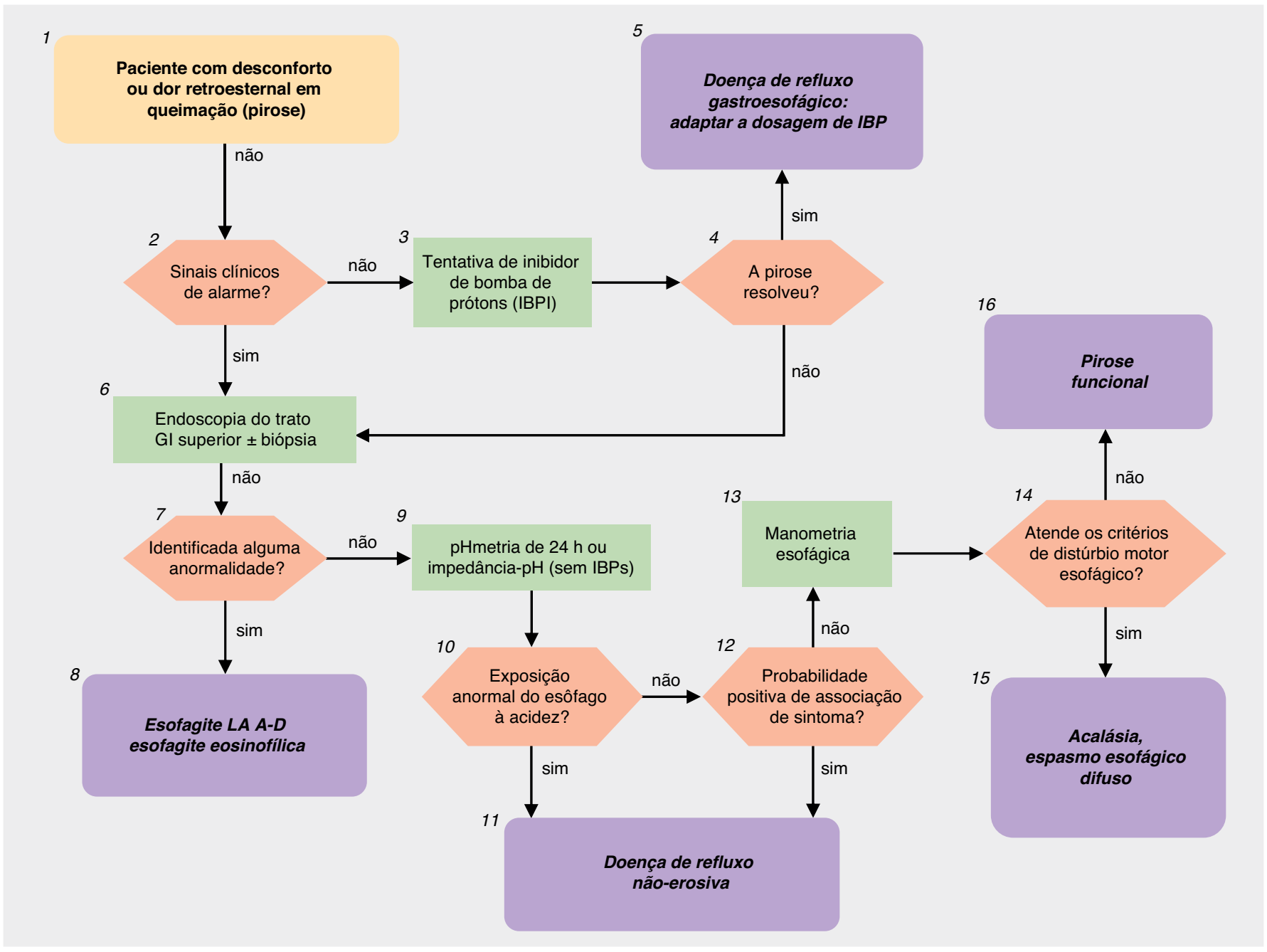

FIGURA 1. Pirose recorrente

Legenda da figura 1

1. Deve-se observar que pirose é descrita de forma bastante diversa entre as diversas culturas e idiomas. A maioria das descrições deste sintoma inclui sensação de desconforto ou queimação atrás do esterno, que pode se irradiar em direção ao pescoço. A pirose é um sintoma intermitente, mais comumente experimentado nos períodos pós-prandiais iniciais, durante o exercício e enquanto deitado em decúbito. Este desconforto é aliviado ao beber água ou tomar um antiácido. O sintoma pode interferir nas atividades normais. A dor ou o desconforto epigástrico não ascendentes para a região retroesternal, não devem ser chamados de pirose ${ }^{(1)}$.

2. A história e o exame físico devem procurar por sinais clínicos de alarme sugestivos de câncer. Isto incluiria evidência de disfagia persistente, sangramento GI, perda de peso não intencional, linfadenopatia, massa epigástrica e evidência de anemia.

3. Não há nenhum padrão fixo para a dose, duração ou medicamento específico a ser usado em uma tentativa de IBP para pirose, o que torna razoável tratar com uma dose padrão 1 vez ao dia por duas semanas ${ }^{(11)}$.

4. Se não for alcançada resposta suficiente com a dose padrão de IBP, esta deve ser aumentada para 2 vezes ao dia por, no mínimo, 2 semanas, antes de considerá-la como falha no tratamento.

5. Uma vez conseguida resposta satisfatória, a dose de IBP deve ser reduzida para a menor quantidade que ainda esteja associada a um efeito satisfatório do tratamento.

6. As biópsias devem ser obtidas no momento da endoscopia, se houver qualquer anormalidade visível, sugestiva de metaplasia ou esofagite eosinofílica, ou se a disfagia for um sintoma adicional presente. Se houver suspeita de esofagite eosinofílica, devem-se obter 5 biópsias da mucosa ${ }^{(12,13)}$. Embora também possam ser detectados os critérios histológicos para esofagite (hiperplasia de células basais, redes de pequenos vasos que se projetam em direção à superfície mucosa), esses achados carecem de especificidade para a doença do refluxo gastroesofágico (DRGE) ${ }^{(14)}$.

7. Anormalidades relevantes, durante a endoscopia do trato GI superior, que excluiriam o diagnóstico de pirose funcional, são a esofagite de refluxo ou a esofagite eosinofílica.

8. A Classificação de Los Angeles de esofagite se baseia na ocorrência e na extensão das rupturas visíveis da mucosa esofágica distal. A forma Los Angeles A é a mais leve com apenas pequenas rupturas $(<5 \mathrm{~mm})$ confinadas às dobras do epitélio, enquanto a Los Angeles D é a mais grave com rupturas quase circunferenciais ${ }^{(15)}$. A esofagite eosinofílica geralmente é atribuível à alergia aos alérgenos ingeridos (alimento) ou inalados e definida pelo achado de $\geq 15$ eosinófilos por campo, em grande aumento nas biópsias da mucosa esofágica ${ }^{(16)}$. 
9. A pHmetria ou a impedância-pH de 24 h é realizada após a suspensão da terapia com IBP por 7 dias, para se obter avaliação significativa da exposição do esôfago ao ácido e proporcionar a melhor possibilidade de se encontrar uma associação positiva entre os episódios de pirose e os eventos de refluxo ${ }^{(17,18)}$.

10. O ponto de corte para a exposição anormal do esôfago ao ácido é geralmente < $5 \%$, embora este valor apresente ligeiras variações entre os centros ${ }^{(19)}$.

11. A definição de Roma III de doença de refluxo não-erosivo é a exposição ácida anormal ou uma associação positiva de sintoma-refluxo na ausência de sinais macroscópicos endoscópicos de esofagite de refluxo ${ }^{(20,21)}$

12. A PAS é um teste estatístico para determinar se a ocorrência simultânea de sintomas e eventos de refluxo em períodos de 2 minutos ocorre ao acaso ou em função dos dois estarem, provavelmente, relacionados. Uma PAS $>95 \%$ equivale a um $P<0,05$ de que eles estão relacionados ${ }^{(22)}$. Apesar de alguns centros usarem o índice de sintoma (IS) para avaliar a associação refluxo-sintoma, o IS não é método validado e não tem base estatística ${ }^{(23)}$.

13. É preferível se obter um estudo de manometria de alta resolução (topografia da pressão esofágica) se estiver disponível, em razão de maior sensibilidade no diagnóstico de acalásia ${ }^{(24)}$.

14. Para o propósito de se estabelecer o diagnóstico de pirose funcional, os dois únicos diagnósticos excludentes são acalásia ou espasmo esofágico difuso (EED). Outras anormalidades peristálticas menos graves, são, contudo, consistentes com diagnóstico de pirose funcional.

15. A acalásia é definida pela ausência de peristalse e pelo relaxamento incompleto do EEI à deglutição; EED por $\geq 20 \%$ das deglutições, apresentando contrações simultâneas ou espásticas no esôfago distal durante o exame ${ }^{(25)}$.

16. Os critérios diagnósticos Rome III para pirose funcional são:

a. desconforto ou dor retroesternal em queimação;

b. ausência de evidência de que o refluxo gastroesofágico é a causa do sintoma;

c. ausência de distúrbios da motilidade esofágica baseados em alterações histopatológicas, e

d. critérios preenchidos por pelo menos 3 meses, com início do sintoma no mínimo 6 meses antes do diagnóstico ${ }^{(26)}$.

\section{DOR TORÁCICA RECORRENTE COM SUSPEITA DE ORIGEM ESOFÁGICA}

\section{Anamnese}

Professora aposentada de 72 anos de idade consulta um gastroenterologista, encaminhada pelo cardiologista. Nos últimos 2 anos experimentou vários episódios de dor retroesternal grave, que se irradia para a mandíbula e para o braço esquerdo; algumas vezes também há irradiação da dor para a linha média das costas (Caixa 1, Figura 2). Antes do início da dor torácica a paciente raramente apresentou problemas de saúde. $\mathrm{O}$ seu apêndice foi removido aos 22 anos de idade e submeteu-se a uma histerectomia aos 52 anos, em razão de fibromas. Não apresenta história familiar relevante de doença gastrointestinal.

A dor no peito ocorre com uma incidência média de dois episódios por semana, com grandes variações na frequência da ocorrência; é descrita como uma sensação opressiva. O início da dor não está claramente relacionado com a ingestão de refeição e não há disfagia entre ou durante os episódios. Não há pirose, regurgitação nem odinofagia típicos. O início da dor não está claramente relacionado ao exercício ou à postura corporal. O exame físico dos pulmões e da parede torácica é normal (Caixa 2). Em três ocasiões a paciente foi internada na unidade coronariana de um grande hospital geral. Não foi encontrada evidência de isquemia miocárdica nem de infarto em nenhuma das ocasiões. A radiografia do tórax é normal. A angiografia coronariana revelou artérias coronárias normais e o teste de esforço foi normal (Caixas 5 e 6 ). Antes do encaminhamento ao gastroenterologista, foi feita tentativa terapêutica com omeprazol $40 \mathrm{mg} 2$ vezes ao dia (Caixa 8). Após 6 semanas deste tratamento, a paciente relatou que a dor no peito continuava ocorrendo (Caixa 9).

O gastroenterologista solicita endoscopia do trato GI superior (Caixa 11) em que é observada junção escamo-colunar normal, posicionada $1 \mathrm{~cm}$ proximal à impressão diafragmática (Caixa 12). Neste estágio, o médico decide solicitar exames adicionais: manometria esofágica (Caixa 18), seguida por $\mathrm{pHmetria}$ de 24 horas, sem uso de inibidor de bomba de próton (Caixa 14). Estes apresentam peristalse normal, função do EEI normal (Caixa 19) e exposição fisiológica ao ácido (tempo com $\mathrm{pH}<4: 3,2 \%$ ) (Caixa 15). Durante o estudo de 24 h não ocorreram episódios de dor no peito. Portanto, não pode ser estabelecida a probabilidade de associação de sintoma (Caixa 17). Conclui-se por diagnóstico de dor torácica funcional com origem esofágica presumida (Caixa 21). 
1

Paciente com dor no peito

recorrente, com suspeita de

origem esofágica

2

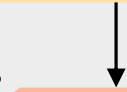

História e exame

físico sugestivos

de etiologia

não esofágica

4 กão

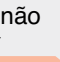

Risco de etiologia cardíaca?
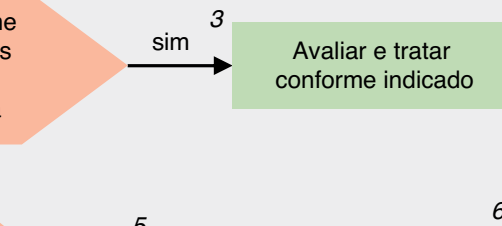<smiles></smiles>
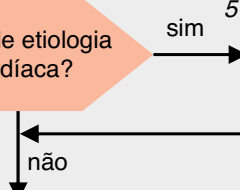

Avaliação
cardiológica

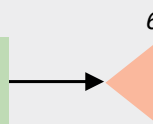

Tentativa com inibidor de
bomba de próton (IBP)

duas vezes ao dia

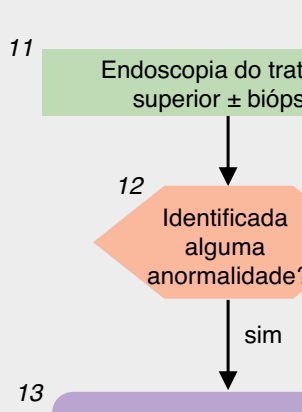

13

Esofagite LA A-D,

esofagite medicamentosa,

esofagite infecciosa
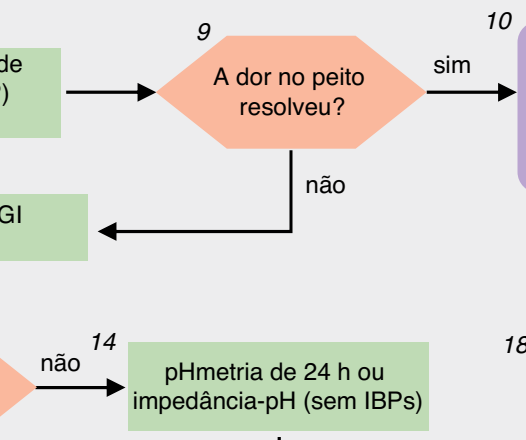

Identificada

alguma

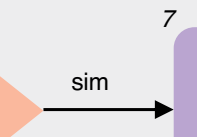
normalidade? não

quêmica, pericardite

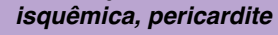

\section{(1)}

Doença de refluxo

com dor no peito:

adpatar a dosagem de IBP

não

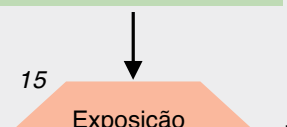

18

Dor torácica funcional

de origem esofágica

presumida

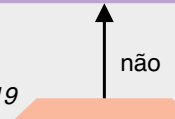

Preenche os

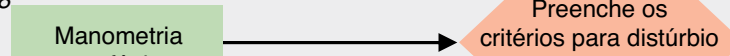

do motor esofágico?

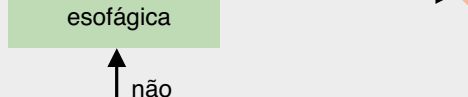

\section{Probabilidade}

anormal do esôfago $\stackrel{\text { não }}{\longrightarrow}$ positiva de associação

de sintoma?
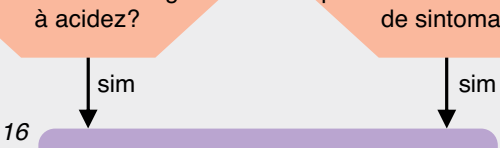

$\operatorname{sim}$

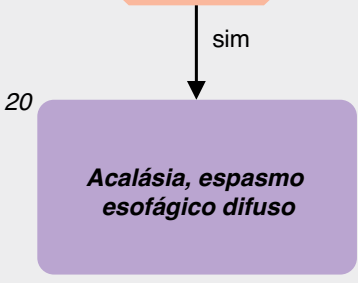

Doença do refluxo não-erosiva

FIGURA 2. Dor torácica recorrente com suspeita de origem esofágica

Legenda da figura 2

1. A dor torácica esofágica é geralmente descrita como retroesternal com irradiação para a linha média das costas. Pode ser uma sensação de opressão e imitar de maneira muito próxima a dor cardíaca. Também pode ocorrer a irradiação para a mandíbula e para o braço esquerdo.

2. O histórico e o exame físico devem buscar evidência de etiologias musculoesquelética, pulmonar ou neurológica da dor torácica.

3. Se for estabelecido um diagnóstico alternativo, que seja tipicamente associado com a dor torácica, concluiria a avaliação para dor torácica esofágica funcional.

4. É importante considerar adequadamente o risco de condições cardíacas potencialmente fatais antes de se prosseguir para uma avaliação esofágica. Esta necessidade nem sempre significa encaminhamento cardiológico, mas se existir a dúvida, é melhor errar para o lado da precaução.

5. A avaliação cardiológica relevante pode incluir o teste de esforço, o monitoramento com Holter e a angiografia coronária, dependendo das características do sintoma e dos fatores de risco(27)

6. Se for estabelecido um diagnóstico cardiológico que seja tipicamente associado com a dor no peito, a avaliação concluiria por dor torácica esofágica funcional.

7. Apesar de outros diagnósticos cardiológicos poderem potencialmente explicar a dor torácica, os dois que apresentam consequências mais imediatas são a doença da artéria coronária e a pericardite.

8. Para o sintoma de dor torácica relacionado à suspeita de refluxo, recomenda-se uma tentativa com IBP duas vezes ao dia por 4 semanas ${ }^{(28)}$.

9. Se a terapia com IBP estiver associada à uma melhora satisfatória ou à resolução da dor no peito, a avaliação concluiria por dor torácica esofágica funcional.

10. Uma vez conseguida resposta satisfatória, a dose de IBP deve ser reduzida para a menor quantidade que ainda esteja associada a efeito satisfatório do tratamento. 
11. As biópsias devem ser obtidas no momento da endoscopia, se houver qualquer anormalidade visível sugestiva de metaplasia, ulceração, infecção, esofagite eosinofílica ou se a disfagia for um sintoma adicional apresentado.

12. Os achados endoscópicos diagnósticos de uma condição esofágica dolorosa concluiriam a avaliação por dor torácica esofágica funcional.

13. A causa mais comum da dor torácica esofágica é a doença de refluxo gastroesofágico ${ }^{(3)}$, mas podem ser encontradas outras causas de ulceração esofágica, como a cáustica, infecciosa ou esofagite induzida por medicamento. A Classificação de Los Angeles de esofagite se baseia na ocorrência e na extensão das rupturas visíveis da mucosa na porção esofágica distal. A forma Los Angeles A é a mais leve com apenas pequenas rupturas (< 5 mm) confinadas às dobras do epitélio enquanto que a Los Angeles D é a mais grave, com rupturas quase circunferenciais ${ }^{(15)}$.

14. A pHmetria de 24 horas ou a impedâncio-pHmetria é realizada após a suspensão da terapia com IBP por 7 dias para se obter uma avaliação significativa da exposição do esôfago ao ácido e proporcionar a melhor possibilidade de encontrar uma associação positiva entre os episódios de pirose e os eventos de refluxo.

15. O ponto de corte para a exposição anormal do esôfago ao ácido é geralmente < $5 \%$, embora este valor apresente ligeiras variações entre os centros ${ }^{(19)}$.

16. A exposição anormal do esôfago ao ácido ou a probabilidade positiva de associação de sintoma, são diagnósticos de doença de refluxo não erosiva pelos critérios de Roma III. Isto então estabeleceria um diagnóstico de síndrome de dor torácica de refluxo (e excluiria a dor torácica funcional).

17. A Probabilidade de Associação de Sintoma (PAS) é um teste estatístico para determinar se a ocorrência simultânea de sintomas e eventos de refluxo, em períodos de 2 minutos, surge ao acaso ou em função dos dois estarem provavelmente relacionados ${ }^{(22)}$. Uma PAS $>95 \%$ equivale a uma $P<0,05$ de que eles estão relacionados. Apesar de alguns centros usarem o Índice de Sintoma (IS) para avaliar a associação refluxo-sintoma, refluxo-sintoma não é um método validado e não tem base estatística ${ }^{(23)}$.

18. Se o refluxo não puder ser considerado como a causa da dor no peito, a manometria esofágica é indicada. É preferível se obter um estudo de manometria de alta resolução (topografia da pressão esofágica) se estiver disponível, em razão de uma maior sensibilidade no diagnóstico de acalásia. ${ }^{(24)}$.

19. Para o propósito de se estabelecer um diagnóstico de dor torácica funcional, os dois únicos diagnósticos excludentes são a acalásia ou o EED. De acordo com os critérios de Roma III, outras anormalidades peristálticas menos graves são também consistentes com um diagnóstico de dor torácica funcional.

20. A acalásia é definida pela ausência de peristalse e pelo relaxamento incompleto do EEI à deglutição; EED por $\geq 20 \%$ das deglutições apresentando contrações simultâneas ou espásticas no esôfago distal durante o exame ${ }^{(25)}$.

21. Os critérios diagnósticos de Roma III para dor torácica funcional de origem presumidamente esofágica são:

1. dor ou desconforto retroesternal que não é em queimação;

2. ausência de evidência de que o refluxo gastroesofágico é a causa do sintoma;

3. ausência de distúrbios da motilidade esofágica baseados em alterações histopatológicas e,

4. critérios atendidos por pelo menos 3 meses, com início do sintoma no mínimo 6 meses antes do diagnóstico ${ }^{(26-29)}$.

\section{DISFAGIA}

\section{Anamnese}

Motorista de ônibus de 44 anos de idade é encaminhado ao gastroenterologista pelo seu médico de atenção primária em função de disfagia para alimentos sólidos e líquidos (Caixa 1, Figura 3). Os sintomas começaram há cerca de 1 ano. Inicialmente eram intermitentes e leves, mas nas últimas semanas a percepção do bolo alimentar trancar ao nível médio-torácico é percebida em quase todas as refeições. Não apresenta dor no peito nem odinofagia, nem há características de disfagia orofaríngea e o exame físico para causas não esofágicas da disfagia é negativo (Caixa 2). Seu peso corporal tem permanecido constante em $92 \mathrm{~kg}$. O paciente experimenta pirose 1 vez por semana em média. Breve tentativa terapêutica com um antagonista do receptor $\mathrm{H}_{2}$ iniciada pelo seu médico de atenção primária, eliminou a pirose, mas não melhorou a disfagia. Sua história médica, por outro lado, é normal e o paciente não relata uso de nenhum medicamento. Não há história familiar de doença gastrointestinal.

Realizou-se endoscopia do trato GI superior (Caixa 4), excluindo-se esofagite macroscópica ou qualquer lesão orgânica que causasse obstrução esofágica (Caixa 5) e o exame microscópico das biópsias obtidas do esôfago distal, assim como do proximal, mostra que não há nenhuma evidência de esofagite eosinofílica ou de outra anormalidade histológica. A deglutição de bário com estimulação com bolo de marshmallow (Caixa 6) não revela lesão estrutural nem comprometimento do trânsito através do esôfago (Caixa 7). Em função da presença de sintomas leves de refluxo (Caixa 9), inicia-se uma tentativa com IBP omeprazol $40 \mathrm{mg}$ duas vezes ao dia (Caixa 10). Isto não resulta em melhora da disfagia do paciente (Caixa 11).

O gastroenterologista então solicita um estudo manométrico do esôfago (Caixa 13), que mostra peristalse esofágica normal, pressão normal do EEI (esfíncter esofágico inferior) e relaxamento normal do EEI após a deglutição (Caixa 14). O monitoramento concomitante da impedância confirma o trânsito completo do bolo em 9 de 10 deglutições, achados que estão dentro da faixa normal. $\mathrm{O}$ paciente nega novamente qualquer sintoma cervical (Caixa 16). Conclui-se pelo diagnóstico de disfagia funcional (Caixa 20). 
1

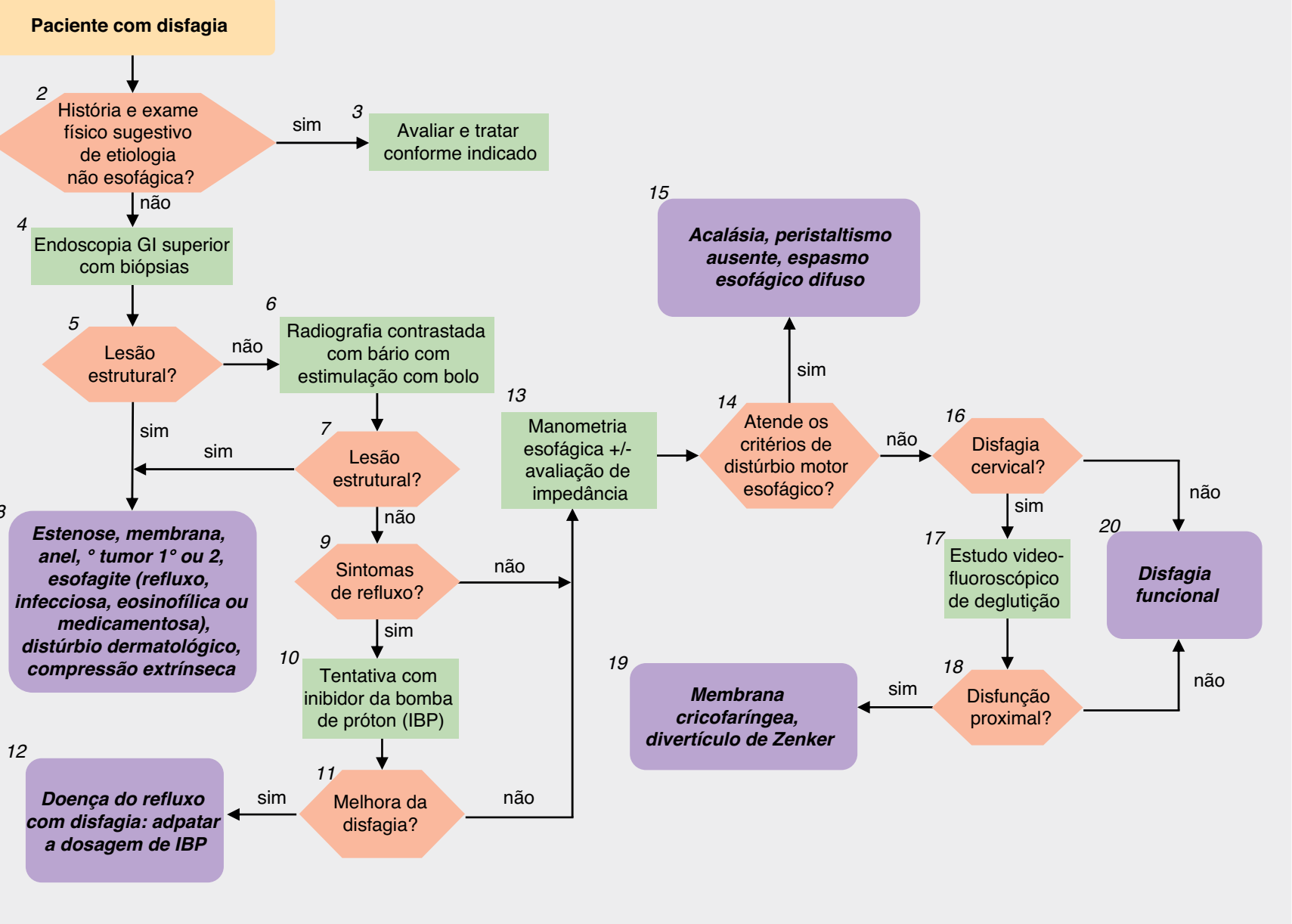

FIGURA 3. Disfagia

Legenda da figura 3

1. A disfagia deve ser caracterizada por sua ocorrência apenas com alimentos sólidos, quando sugere uma anormalidade estrutural, ou com ambos sólidos e líquidos, sugerindo anormalidade de motilidade e pela sua localização, proximal ou distal. Também é importante o sintoma associado de odinofagia, pois este é altamente sugestivo de ulceração esofágica.

2. A história e o exame físico devem explorar as causas não-esofágicas da disfagia: massas no pescoço, bócio ou achados neurológicos compatíveis com o diagnóstico de disfagia orofaríngea.

3. A detecção de uma condição não-esofágica associada à disfagia concluiria a avaliação para disfagia funcional.

4. As biópsias devem ser obtidas no momento da endoscopia, independentemente de anormalidades visíveis, para avaliar esofagite eosinofílica; devem ser obtidas cinco biópsias da mucosa ${ }^{(12-13)}$. Embora também possam ser detectados os critérios histológicos para esofagite (hiperplasia de célula basal, pregas do plexo prolongando em direção à superfície), esses achados carecem de especificidade para doença do refluxo gastroesofágico (DRGE) ${ }^{(14)}$.

5. A detecção de lesão estrutural concluiria a avaliação para disfagia funcional.

6. A deglutição de bário com estimulação do bolo sólido (comprimido de bário ou marshmallow impregnado com bário) é útil na detecção de lesões obstrutivas, como um discreto anel no esôfago distal. Nesta aplicação, este exame possui sensibilidade superior à da endoscopia do trato GI superior $^{(30)}$

7. A detecção de lesão estrutural concluiria a avaliação para disfagia funcional.

8. A disfagia é um sintoma comum de uma multiplicidade de distúrbios esofágicos inflamatórios e estruturais e a deteç̧ão destes excluiria a disfagia funcional ${ }^{(31-33)}$.

9. Sintomas concomitantes de pirose e regurgitação sugerem que a DRGE pode ser a causa da disfagia.

10. Ao ser usado como tentativa terapêutica em disfagia, os IBPs normalmente são administrados em regime de duas vezes ao dia por, no mínimo, 2 semanas $^{(34)}$.

11. A resolução da disfagia com a terapia IBP concluiria que a mesma foi uma manifestação da doença de refluxo e exclui o diagnóstico de disfagia funcional.

12. Como ocorre com todos os pacientes, uma vez estabelecida resposta satisfatória ao tratamento, a dose de IBP deve então ser reduzida à dose mínima, que ainda associe uma resposta satisfatória ao tratamento. 
13. Se não for encontrada nenhuma anormalidade estrutural, é indicada a manometria. Pode ser preferível se obter estudo de manometria de alta resolução (topografia da pressão esofágica) se estiver disponível, em razão de uma maior sensibilidade no diagnóstico de acalásia e de outros distúrbios de motilidade ${ }^{(35-36)}$. Se disponível, a avaliação concomitante do trânsito esofágico, com base na impedância, pode fornecer informações adicionais com relação ao trânsito do bolo no esôfago ter se completado integralmente.

14. Os critérios de Roma III estipulam que distúrbios da motilidade esofágica baseados em alterações histopatológicas, espasmo esofágico difuso (EED) e acalásia excluem o diagnóstico de disfagia funcional. A acalásia é definida pela peristalse ausente e pelo relaxamento incompleto do EEI, em resposta à deglutição; o EED define-se pela presença de $\geq 20 \%$ das contrações simultâneas ou espásticas no esôfago distal durante a realização de deglutições líquidas (habitualmente são realizadas 10 deglutições) ${ }^{(25)}$.

15. Propõe-se que, além do EED e da acalásia, a peristalse ausente ou gravemente interrompida, também deva resultar em exclusão do diagnóstico de disfagia funcional.

16. A disfagia alta (cervical, orofaríngea) é relatada por cerca de $30 \%$ dos indivíduos com doença distal. Entretanto, como a doença distal foi adequadamente excluída, a sugestão de qualquer evidência de disfagia cervical deve induzir à avaliação para disfunção esofágica proximal.

17. Estudo videofluoroscópico de deglutição permite um exame detalhado do mecanismo de deglutição, incluindo as características de abertura do esfíncter esofágico superior que, com freqüência, é uma área cega na endoscopia ou no exame de deglutição de bário, em função da falta de distensão adequada $^{(37)}$. Observe que, se o estudo inicial com bário (Quadro 6) incluiu a videofluoroscopia, como ocorre em algumas instituições, esta etapa pode ser omitida.

18. O achado de disfunção esofágica proximal encerraria a avaliação para disfagia funcional.

19. Membrana cricofaríngea é provocada pela degeneração fibrosa no esfíncter esofágico superior, resultando em abertura restrita e pode ser aceita como a causa da disfagia, uma vez excluídas outras doenças ${ }^{(38)}$. Isto também pode resultar na formação do divertículo de Zenker, cuja boca está localizada bem próxima ao cricofaríngeo.

20. Os critérios diagnósticos de Roma III para disfagia funcional são:

a. a percepção da passagem dos alimentos, líquidos ou sólidos, ao longo do esôfago. Na linguagem do paciente, pode ser referida como alimento que tranca, fica retido ou passa com dificuldade;

b. ausência de evidência de que o refluxo gastroesofágico é a causa do sintoma;

c. ausência de distúrbios da motilidade esofágica baseados em alterações histopatológicas, e

d. critérios preenchidos por pelo menos 3 meses, com início do sintoma no mínimo 6 meses antes do diagnóstico ${ }^{(26)}$.

\section{SENSAÇÃO DE BOLO NA GARGANTA}

\section{Anamnese}

Empresária de um pequeno negócio, de 30 anos de idade, consulta seu médico de atenção primária (MAP) em razão de sensação de bolo na garganta (Quadro 1, Figura 4). Ela tem apresentado este sintoma de forma intermitente por cerca de 1 ano, mas a intensidade tem aumentado nas últimas semanas. Não há nenhum evento desencadeador óbvio. Sua deglutição é normal e sem dor. Não há disfagia (Quadro 4) nem odinofagia e ela não apresenta rouquidão nem qualquer outra alteração em sua voz (Quadro 6). O sintoma melhora ao se alimentar. Mesmo assim a paciente tem a impressão de que alguma coisa está parada na sua garganta. Raramente tem pirose e não perdeu peso. Exceto por isto, a paciente está sadia. Não fuma e ingere álcool com moderação (Quadro 6). Com exceção de anticoncepcional oral, ela não faz uso de nenhum medicamento.

O MAP examina o pescoço, a garganta e a cavidade oral da paciente e não encontra anormalidades (Caixa 2). Não há massas palpáveis, nem linfonodos ou tireoide aumentados. Tem-se a impressão de que a paciente está experimentando alguma ansiedade e verifica se a sensação de bolo está temporalmente relacionada ao estresse, mas a paciente nega esta associação. Convencido de que está lidando com um distúrbio funcional, o MAP explica à paciente que não há nada seriamente errado e tenta tranquilizá-la.

Entretanto, 2 semanas mais tarde a paciente consulta novamente o médico em função da continuidade da sensação de bolo. É então, encaminhada a um otorrinolaringologista, pois revendo sua história, tivera exposição substancial a fumo passivo e estava bastante preocupada com isto (Caixa 7). O especialista não encontrou nenhuma anormalidade no exame que incluiu a nasolaringoscopia (Caixa 8). Por existir uma associação entre o globus e o achado endoscópico de mucosa gástrica ectópica no esôfago proximal e o câncer esofágico, a paciente é encaminhada para endoscopia (Caixa 10). Não foram encontradas anormalidades (Caixa 11). Iniciou-se tentativa de tratamento de 6 semanas com IBP em função da presença de alguma pirose (Caixa 14). Isto não resultou em melhora da sensação de bolo (Caixa 15). A paciente é novamente tranquilizada sobre a natureza benigna da sua condição e é feito um diagnóstico de globus (Caixa 17). 


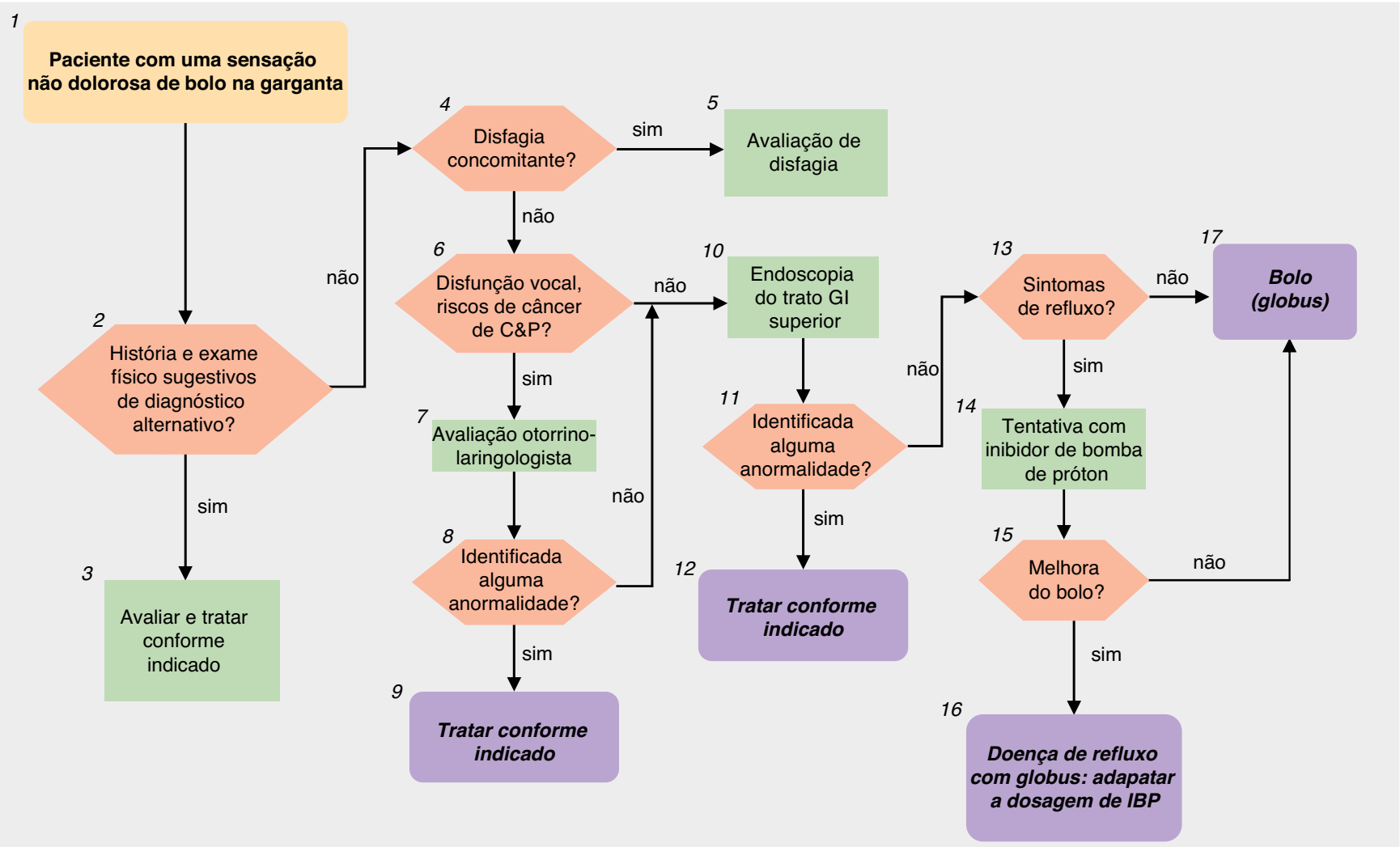

FIGURA 4. Sensação de bolo na garganta

\section{Legenda da figura 4}

1. A sensação de globus (palavra grega para "bola") é a sensação de um bolo ou "bola" na garganta, diferente da disfagia, que é experimentada sem deglutir e até mesmo apresenta melhora ao deglutir; não é dolorosa.

2. A história e o exame físico, especialmente do pescoço, da garganta e da cavidade oral, podem revelar evidência de trauma ou condição inflamatória ou outra que explique potencialmente os sintomas.

3. A descoberta de um diagnóstico alternativo suficiente para explicar o sintoma impediria o diagnóstico de globus.

4. A sensação de globus pode ocorrer em associação com a disfagia, caso em que os esforços diagnósticos são alterados para avaliação de disfagia.

5. As condições associadas à disfagia impediriam um diagnóstico de globus funcional; ver o algoritmo para disfagia.

6. A sensação de globus pode ocorrer em associação com outros sintomas da disfunção laríngea, como a rouquidão, que deve resultar em avaliação do otorrinolaringologista (OTR). Da mesma forma, se um paciente apresenta o risco de câncer na laringe em função de tabagismo, ele ou ela deve fazer uma avaliação de OTR ${ }^{(39)}$.

7. A avaliação do OTR incluiria provavelmente a nasolaringoscopia e outros exames de imagem, conforme indicado ${ }^{(40,41)}$.

8. A identificação de uma anormalidade na avaliação do OTR impediria o diagnóstico de globus funcional.

9. As outras condições de OTR resultariam em tratamento, conforme indicado.

10. A endoscopia é realizada para avaliar a mucosa gástrica ectópica no esôfago cervical ou o câncer esofágico, que pode estar associado à sensação de globus.

11. A identificação de uma anormalidade na endoscopia concluiria a avaliação para globus funcional. O achado de mucosa gástrica ectópica no esôfago proximal deve resultar na consideração de terapia de ablação.

12. As outras condições devem ser tratadas conforme indicado.

13. A doença de refluxo pode ser a causa do globus e a maioria dos pacientes de DRGE não apresentará achados endoscópicos macroscópicos. Portanto, os pacientes com sintomas como pirose ou regurgitação devem se submeter a uma tentativa terapêutica de tratamento antirrefluxo.

14. Quando usados como tentativa terapêutica em globus, os IBPs, em geral, são administrados em regime de 2 vezes ao dia ${ }^{(42)}$.

15. A resolução do globus com a terapia IBP concluiria que este seria uma manifestação da doença de refluxo e excluiria o diagnóstico de globus funcional.

16. Uma vez estabelecida resposta satisfatória ao tratamento, a dose de IBP deve, então, ser reduzida à dose mínima que ainda associe resposta satisfatória ao tratamento.

17. Os critérios diagnósticos Rome III para globus são:

a. sensação persistente ou intermitente, não-dolorosa de um bolo ou de corpo estranho na garganta;

b. ocorrência da sensação entre as refeições;

c. ausência de disfagia ou de odinofagia;

d. ausência de evidência de que o refluxo gastroesofágico seja a causa do sintoma

e. ausência de distúrbios da motilidade esofágica baseados em alterações histopatológicas;

f. critérios atendidos por pelo menos 3 meses com início do sintoma no mínimo 6 meses antes do diagnóstico ${ }^{(26)}$. 


\section{CONFLITO DE INTERESSE}

Garantia do artigo: Rome Foundation.

Contribuições específicas dos autores: Peter J. Kahrilas: conceito e desenho, análise e interpretação dos dados, rascunho do artigo.

André J. P. M. Smout: coautoria.

Apoio financeiro: Financiado com a subvenção da Rome Foundation.

Potenciais conflitos de interesse: Nenhum.

\section{REFERÊNCIAS}

1. Vakil N, Zanten van SV, Kahrilas P, et al. The Montreal definition and classification of gastroesophageal reflux disease: a global evidence-based consensus Am J Gastroenterol. 2006;101:1900-20.

2. Tytgat GN, McColl K, Tack J, et al. New algorithm for the treatment of gastro-oesophageal reflux. Aliment Pharmacol Ther. 2008;27:249-56.

3. Voskuil JH, Cramer MJ, Breumelhof R, et al. Prevalence of esophageal disorders in patients with chest pain newly referred to the cardiologist. Chest. 1996;109:1210-4.

4. Sengupta JN. An overview of esophageal sensory receptors. Am J Med. 2000;108:87S-9S

5. Broekaert D, Fischler B, Sifrim D, et al. Influence of citalopram, a selective serotonin reuptake inhibitor, on oesophageal hypersensitivity: a doubleblind, placebo-controlled study. Aliment Pharmacol Ther. 2006;23:365-70.

6. Rao SS, Mudipalli RS, Remes-Troche JM, et al. Theophylline improves esophageal chest pain - a randomized, placebo-controlled study. Am J. Gastroenterol 2007;102:930-8.

7. Chen CL, Orr WC. Comparison of esophageal motility in patients with solid dysphagia and mixed dysphagia. Fall. 2005;20:261-5.

8. Ali $\mathrm{KH}$, Wilson JA. What is the severity of globus sensation in individuals who have never sought health care for it? J Laryngol Otol. 2007;121:865-8.

9. Deary IJ, Wilson JA, Kelly SW. Globus pharyngis, personality, and psychological distress in the general population. Psychosomatics. 1995;36:570-7.

10. Locke GR III, Talley NJ, Fett SL, et al. Prevalence and clinical spectrum of gastroesophageal reflux: a population-based study in Olmsted County, Minnesota. Gastroenterology. 1997;112:1448-56.

11. Howden CW, Chey WD. Gastroesophageal reflux disease. J Fam Pract 2003; $52: 240-7$.

12. Gonsalves N, Policarpio-Nicolas M, Zhang Q, et al. Histopathologic variability and endoscopic correlates in adults with eosinophilic esophagitis. Gastrointest Endosc. 2006;64:313-9.

13. Kapel RC, Miller JK, Torres C, et al. Eosinophilic esophagitis: a prevalent disease in the United States that affects all age groups . Gastroenterology. 2008;134:1316-21.

14. Vieth M. Structural abnormalities of endoscopy-negative reflux disease-real or perceived? Digestion. 2008;78:24-30.

15. Lundell LR, Dent J, Bennett JR, et al. Endoscopic assessment of oesophagitis: clinical and functional correlates and further validation of the Los Angeles Classification. Gut. 1999;45:172-80.

16. Moawad FJ, Veerappan GR, Wong RK. Eosinophilic esophagitis. Dig Dis Sci. 2009;54:1818-28.

17. Savarino E, Zentilin O, Tutuian $\mathrm{R}$ et al. The role of nonacid reflux in NERD lessons learned from impedance-pH monitoring in 150 patients off therapy. Am J Gastroenterol. 2008;103:2685-93.
18. Hemmink GJM, Bredenoord AJ, Weusten BLAM, et al. Esophageal pHimpedance monitoring in patients with therapy-resistant reflux symptoms: "on" of "off" proton pump inhibitor? Am J Gastroenterol. 2008;103:2446-53.

19. Richter JE. Ambulatory esophageal pH monitoring. Am J Med. 1997;103:130S-4S

20. Winter JW, Heading RC. The nonerosive reflux disease-gastroesophageal reflux disease controversy. Curr Opin Gastroenterol. 2008;24:509-15.

21. Hershcovici T, Zimmerman J. Functional heartburn vs non-erosive reflux disease: similarities and differences. Aliment Pharmacol Ther. 2008;27:1103-9.

22. Weusten BLAM, Roelofs JMM, Akkermans LMA, et al. The symptom-association probability: an improved method for symptom analysis of 24-hour esophageal ph data. Gastroenterology. 1994;107:1741-5.

23. Wiener GJ, Richter JE, Copper JB, et al. The symptom index: a clinically important parameter of ambulatory 24-hour esophageal $\mathrm{pH}$ monitoring. Am J Gastroenterol. 1988;83:358-61.

24. Pandolfino JE, Kwiatek MA, Nealis T, et al. Achalasia: a new clinically relevan classification by high-resolution manometry. Gastroenterology. 2008;135:1526-33.

25. Spechler SJ, Castell DO. Classification of oesophageal motility abnormalities. Gut. 2001;49:145-51.

26. Galmiche JP, Clouse RE, Balint A, et al. Functional esophageal disorders Gastroenterology. 2006;130:1459-65.

27. Fenster PE. Evaluation of chest pain: a cardiology perspective for gastroenterologists. Gastroenterol Clin North Am. 2004;33:35-40.

28. Wang WH, Huang JQ, Zheng GF, et al. Is proton pump inhibitor testing an effective approach to diagnose gastroesophageal reflux disease in patients with noncardiac chest pain? Arch Intern Med. 2005;165:1222-8.

29. Mudipalli RS, Remes-Troche JM, Andersen L, et al. Functional chest pain: esophageal or overlapping functional disorder. J Clin Gastroenterol. 2007;41:264-9.

30. Ott DJ, Kelley TF, Chen MY, et al. Evaluation of the esophagus with a marshmallow bolus: clarifying the cause of dysphagia. Gastrointest Radiol. 1991;16:1-4.

31. Mackenzie SH, Go M, Chadwick B, et al. Eosinophilic oesophagitis in patients presenting with dysphagia - a prospective analysis. Aliment Pharmacol Ther 2008:28:1140-6.

32. Prasad GA, Talley NJ, Romero Y, et al. Prevalence and predictive factors of eosinophilic esophagitis in patients presenting with dysphagia: a prospective study. Am J Gastroenterol. 2007; 102:2627-32.

33. Müller S, Pühl S, Vieth M, et al. Analysis of symptoms and endoscopic fi ndings in 117 patients with histological diagnoses of eosinophilic esophagitis. Endoscopy. 2009;39:339-44.

34. Kahrilas PJ, Shaheen NJ, Vaezi MF, et al. American Gastroenterological Association Medical Position Statement on the management of gastroesophageal reflux disease. Gastroenterology 2008;135:1383-91.

35. Fox MR, Bredenoord AJ. Oesophageal high-resolution manometry: moving from research into clinical practice. Gut. 2008;57:405-23.

36. Pandolfino JE, Fox MR, Bredenoord AJ, et al. High-resolution manometry in clinical practice: utilizing pressure topography to classify oesophageal motility abnormalities. Neurogastroenterol Motil 2009;21:796-806.

37. Langmore SE. Evaluation of oropharyngeal dysphagia: which diagnostic tool is superior? Curr Opin Otolaryngol Head Neck Surg. 2003;11:485-9.

38. Dantas RO, Cook IJ, Dodds WJ, et al. Biomechanics of cricopharyngeal bars. Gastroenterology. 1990;99:1269-74

39. Harar RP, Kumar S, Saeed MA, et al. Management of globus pharyngeus: review of 699 cases. J Laryngol Otol. 2004;118:522-7.

40. Chung JY, Levine MS, Weinstein GS, et al. Globus sensation: findings on videof uoroscopic examinations. Can Assoc Radiol J. 2003;54:35-40.

41. Takwoingi YM, Kale US, Morgan DW. Rigid endoscopy in globus pharyngeus: how valuable is it? J Laryngol Otol. 2006;120:42-6.

42. Sinn DH, Kim JH, Son HJ, et al. Response rate and predictors of response in a short-term empirical trial of high-dose rabeprazole in patients with globus. Aliment Pharmacol Ther. 2008;27:1275-81. 